\title{
Reducing Dimensions of the Feature Vector of an Image Based on Blocking-DCT
}

This paper was downloaded from TechRxiv (https://www.techrxiv.org).

\section{LICENSE}

CC BY 4.0

SUBMISSION DATE / POSTED DATE

$11-10-2021 / 12-10-2021$

\section{CITATION}

Torkamani Azar, Farah (2021): Reducing Dimensions of the Feature Vector of an Image Based on BlockingDCT. TechRxiv. Preprint.

https://www.techrxiv.org/articles/preprint/Reducing_Dimensions_of_the_Feature_Vector_of_an_Image_Based_( DCT/16782523

$\mathrm{DOI}$

10.36227/techrxiv.16782523.v1 


\title{
Reducing Dimensions of the Feature Vector of an Image Based on Blocking-DCT
}

\author{
Farah Torkamani Azar
}

\begin{abstract}
The number of the image feature elements is a great challenge in image processing. Since the size of images has increased significantly due to new technologies, it is necessary to utilize it to balance its dimensions for subsequent processing so that it integrates all the characteristics of the signal along with its distinguishing indicators from other similar signals. In the present article, to facilitate the solution, we pay attention to the use of DCT transform on image blocks. In a way, we decrease the size of the indicators to $1 / 64$ of the main dimensions, but its ability to compare the quality of images and identify their complexity is still significant.
\end{abstract}

Index Terms-DCT Transform, image processing, feature extraction, image quality, image compression.

\section{INTRODUCTION}

$\mathbf{O}$ NE the vital problems in the field of image and video processing is a Quality assessment of digital image/video. The feature extraction could be used for some applications such as clustering, biomedical field, pattern analysis in images and so on [1]-[5]. Different methods have been defined which are in statistical or transform approaches, graph, learning and entropy. However, we prefer to use features that, while having a significant amount of information, also take up less memory and use less bandwidth if needed to transmission. The use of one 2-D transformations is usually not extensively because of the large conversion matrices, eventhough the statistics of transform coefficients are used. Here we want to use 2-D. Discrete Cosine Transform with respect to the asymmetric properties of its coefficients. In the following sections, the proposed idea and some experiments to invoke the potential of the defined feature vectors are described in sections II and III. Finally, conclusion would be followed in section IV.

\section{The Proposed IDEA}

It is believed that in the image processing field, image structure is a very important base. Parameters of mean, variance, or histogram values are not unique characters of an image. So, we decided to explain an idea to reduce the number of coefficients and simultaneously, preserve the whole color image attributes. It is usually prefer to use a colored space other than $R_{-} G_{-} B$ such as $Y_{-} C b_{-} C r$, because the lighting component which is predominant than the other channels, separated from other components. In this way, the strategy of JPEG compression method which two color channels $\mathrm{Cb}$ and $\mathrm{Cr}$ are used after down-sampling at a rate of 2 or 4 ,

F. Torkamani Azar was with the Department of Electrical Engineering (Electronic and Communication), Shahid Beheshti University, Tehran, Iran e-mail: (f-torkamani@sbu.ac.ir). could be repeated in color feature vectors, also. Since image dimensions that is so large, if the whole image is considered at once, the features in detail could not be accurately analyzed. Therefore, using the image patches $r \times r \times 3$ could be more valuable. However, it should be considered that we have a trade-off on chosen $r$ value. Small value could help us to have a simple computation, although the clear patterns do not appear carefully. In the large patches, patterns could be observed clearly, however, computation complexity increased exponentially. We used $r=64$ in simulations as the suitable value. Let's us to using $P^{j}, \quad j=1,2,3$ as DCT transform of each patch $f$ in one channel. The Distribution density of the values in matrix transform $P$ and their locations represent an outline view of the considered patch $f$.

\section{A. Prepare Features}

For each patch $r \times r \times 3$, three DCT transform matrix as $P^{j}, \quad j=1,2,3$ in size $r \times r$ could be computed. Two approaches based on the valency of DCT coefficients are offered to reduce the dimensions which applying to all three transform matrices:

1. First idea is considering the significant of DCT coefficients in rectangular paths as Fig. 1a that shows a selected part of paths for example. In a matrix in size $r \times r$, there are $r$ paths like Fig.1a. The vector character of Reflective ${ }^{1}$ in Rectangular paths $\left(R_{R}\right)$ in size $1 \times r$ could be obtained as:

$$
\begin{aligned}
& R_{R}^{j}(k)=\Sigma_{(m, n) \epsilon k^{t h} \text { path }}\left|P^{j}(m, n)\right| ; \\
& \text { for } \quad k=1, \cdots, r, \quad j=1,2,3 .
\end{aligned}
$$

Indeed, sum of the absolute values in each path is considered as a recognition character. Using absolute values of matrix $P$ is because of the worthiness of the weight magnitude. Therefore, for each color patch, there are three $1 \times r$ vectors and the number of recognition characters would be $3 r$, or $1 / r$ the whole image.

2. Second approach is based on the diagonal paths as Fig.1b that shows a few of paths for demonstration. So, sum of the absolute values in each diagonal path is considered as a recognition character vector in size $1 \times(2 r-1)$ as:

$$
\begin{aligned}
& D^{j}(k)=\Sigma_{(m, n) \mid k=m+n-1}\left|P^{j}(m, n)\right| ; \\
& \text { for } \quad k=1, \cdots, 2 r-1, \quad j=1,2,3 .
\end{aligned}
$$

In this case, three recognition vector in size $1 \times(2 r-1)$ are used instead of one color patch in size $r \times r \times 3$. However, we

\footnotetext{
${ }^{1}$ Since DCT coefficients are the reflective of image patch on DCT basis images, we used Reflective name.
} 


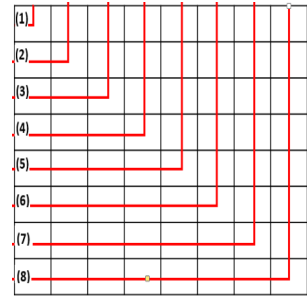

(a)

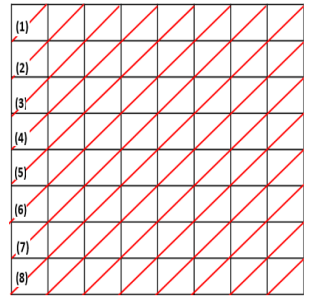

(b)
Fig. 1: (a) The rectangular paths, and (b) diagonal paths in matrix reflection $P$.

suggested to reduce the size of Eq. 2 and called this method as Reflective in Diagonal paths $\left(R_{D}\right)$ :

$$
\begin{aligned}
& R_{D}^{j}(1)=D^{j}(1) ; \\
& R_{D}^{j}(k)=D^{j}(2(k-1))+D^{j}(2 k-1) ; \\
& \text { for } \quad k=2, \cdots, r, \quad j=1,2,3 .
\end{aligned}
$$

In this way, size of three recognition vectors would be $1 \times r$ for each patch image in each component like first approch.

In both outlines, if color components $\mathrm{Cb}$ and $\mathrm{Cr}$ like as JPEG compression are downsampled, there would be one vector in size $1 \times r$ and two vector in size $1 \times(r / 2)$ or two vector in size $1 \times(r / 4)$. It is clear that element number $(1,1)$ of matrices $P^{j}$ is the most significant position which give the mean value of image patch in each channel. The magnitude of element $(1,1)$ is too larger than the other ones. Other important point is that large indexes of $R_{R}$ or $R_{D}$ also indicate the properties of image patch in high frequencies. To demonstrate the potencial ability of distinguishing between variety images by the presented idea, we first consider three examples of an original, noisy and blurry patch samples Fig. 2. $R_{R}$ 's(Eq. 1) and $R_{D}$ 's (Eq. 3) in three components $Y, C b$ and $C r$ of color pace $\mathrm{YCbCr}$ of sample patches 2 to compare the effect of degradation on the feature vectors are shown in Fig. 2d- $2 \mathrm{i}$. As seen on, in all three components, the reflected values of the noisy fragment are higher than the original fragment due to the greater detail and frequency, especially at larger indices that usually there are very small coefficients in the original image. Against, the DCT values of the opaque fragment are smaller than the original patch due to the absence of high frequencies. The first point is that the proposed idea can identify the type of destruction (or the predominant type) as blurring or making noise. The second tip is that sampling from $D$ (Eq. 2) does not reduce the recognition performance. The third matter is that in all three components in the three image samples, $R_{D}$ in last indices are substantially decreasing.

Other samples of patches are selected from VCL database [6]. Three different texture patches from fig. 3 are picked out that shown in three color box: red, magenta and cyan as named No. 1,2 , and 3 respectively. The feature vectors $R_{R}$ and $R_{D}$ of each color components are calculated for three patches Figs. $3 \mathrm{~b}-3 \mathrm{~d}$ to see if these identifier vectors can show the differences in content and psttern of the images, Figs. 4a-4f. As shown in Fig. 4, image samples have various attitude, No.1 is a uniform area, No.3 is a crowded area with so many details that we

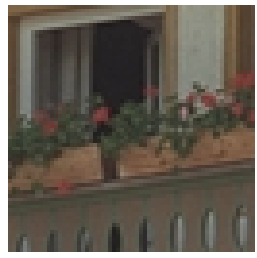

(a)

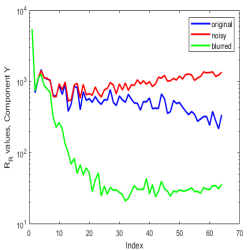

(d)

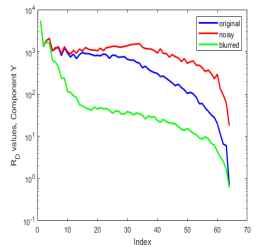

(g)

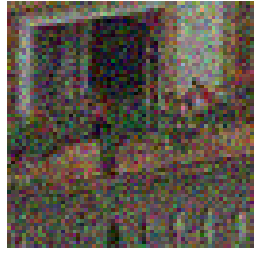

(b)

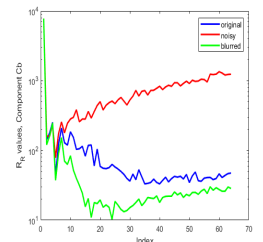

(e)

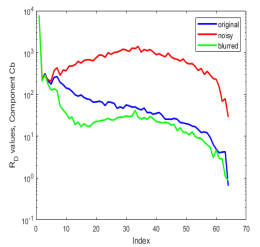

(h)

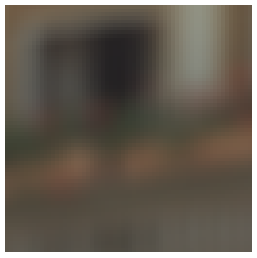

(c)

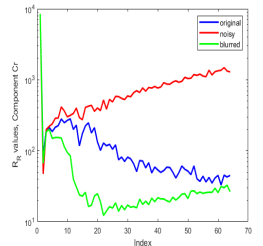

(f)

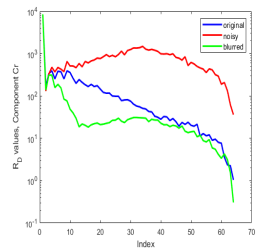

(i)
Fig. 2: (a) original patch, (b) noisy patch, (c) blurry patch, (d) $R_{R}$ values of $Y$ componet, (e) $R_{R}$ values of $C b$ componet, (f) $R_{R}$ values of $C r$ componet, (g) $R_{D}$ values of $Y$ componet, (h) $R_{D}$ values of $C b$ componet, (i) $R_{D}$ values of $C r$ componet.

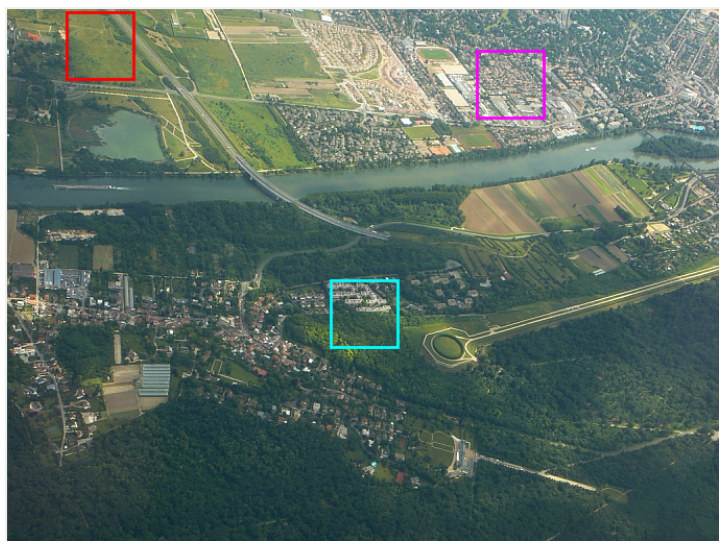

(a)

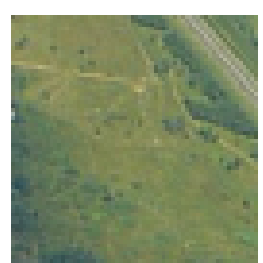

(b)

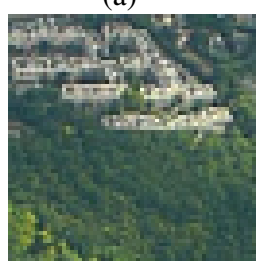

(c)

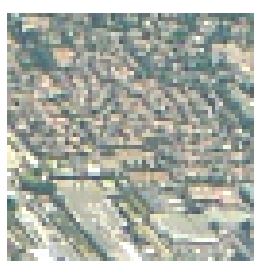

(d)
Fig. 3: One sample image with three selected patches with different patterns, from left to right, No.1-3. 


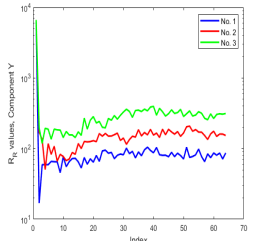

(a)

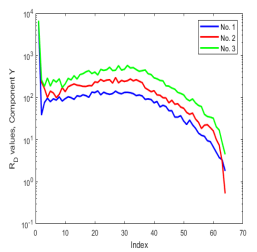

(d)

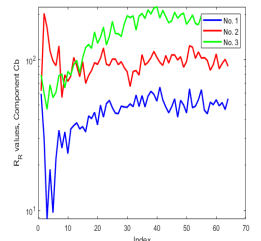

(b)

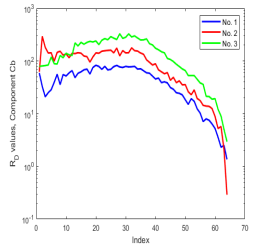

(e)

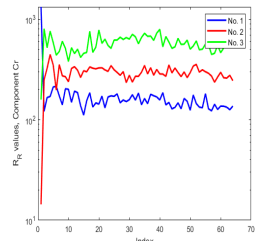

(c)

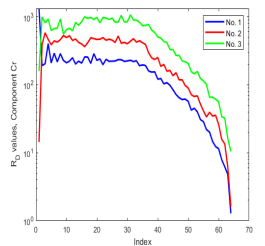

(f)
Fig. 4: first row: $R_{R}$ of three componets $Y, C b$ and $C r$, second row: $R_{D}$ of three channels, left column: $Y$, middle column: $C b$ and right column: $\mathrm{Cr}$ components for three patches of Fig. 3 .

want to see all as much as possible. However, complexity of No. 2 patch is between No.1 and No.3. As can be seen in the feature vectors, the curves of No.1 to 3 are in order. Specifically, No. 1 with a uniform plate have significant DCT values in low indices (low frequencies). With the increasing complexity of patches in the space domain, the feature vector values in higher indices still are valuable. Another noteworthy point is that the vector $R_{D}$, as noted before, due to the sum of the diameters after index $r / 2$ changes with a drastically slope to zero, which is due to the insignificance of the lower half of the matrix of coefficients DCT. For this reason, it seems that identifier vectors $R_{R}$ can be better differentiated.

We presented two types of the feature vectors for each image patch. For each color space component, there is one matrix $K \times r$, with each row being the conversion or reflection values of a $r \times r$ block. Therefore, for each $M \times N \times 3$ color image, a $K \times r \times 3$ tensor can be provided where $K=M N / r^{2}$. The number of values to compare or analyze each color image is reduced to $1 / r$ relative to the original image. However, in the case of colorspace like $\mathrm{YCbCr}$, each patch in component $\mathrm{Cb}$ and $\mathrm{Cr}$ could be downsampled by 2 or 4 and then feature vectors extracted. The important point in comparing the two feature vectors is that the rectangular path is more appropriate than the diagonal path. This is because the diagonal path for the upper indices only calculates the values in position lowright, all of which are close to zero.

\section{EVAluation OF FEATURES}

One of the vital problems in the field of image and video processing is feature extraction of digital image/video. In this section, we tend to simulate two applications for using these features and evaluate their ability criteria.

1.The first one is the image quality measurement in reduced reference mode, where only indicators of the original image are sent to the destination via a secure channel to be used for comparison with the observed image, beyond any doubt, the fewer, the better for both sending and storing. The most comparison methods use mean square differences of features between the original and the degraded images. We have provided for one original image in each component via secure channel, one matrix $K \times r$ where $K$ is the number of used patches in image to extraction features. In receiver, the procedure of feature extraction is repeated for the degraded available image. To compare, the following equations $Q_{R}$ and $Q_{D}$ are introduced. Since comparison steps are the same, consider $A^{j}$ and $B^{j}$ matrices as feauture matrix for original and degraded images in $j^{\text {th }}$ component, regardless they are $R_{R}$ or $R_{D}$.

i-All values in $A^{j}$ has the same significant regardless their position in vector, so the relative difference would be valuable. It should not be forgotten that we are measuring the degraded image respect to one reference.

$$
D^{j}(k, l)=\left|\frac{A^{j}(k, l)-B^{j}((k, l)}{A^{j}((k, l)}\right| ;\left\{\begin{array}{c}
j=1,2,3 ; \\
k=1, \cdots, K ; \\
l=1, \cdots, r ;
\end{array}\right.
$$

where $A^{j}(k, l)$ and $B^{j}(k, l)$ are the $l$ th element of feature vector of $k$ th image patch of the original and degraded images, respectively. $D^{j}$ is the relative difference matrix ${ }^{2}$ which each row belongs to one image patch.

ii-Norm $l_{1}(\cdot)$ of row vector is obtained as the criterion of each patch of the image.

$$
d^{j}(k)=\sum_{l=1}^{r} D^{j}(k, l) / r ; \quad k=1, \cdots, K
$$

According to the footnote, by dividing by $r$ in Eq. 5, we intend to guarantee the upper limit of Eq. 5 to one. However, sometimes the amount of damage to the image is so great that it exceeds one. We keep the same numbers for sorting images by the degree of degradation.

iii-The median value of $d^{j}$ related to the whole image is considered as the image quality measurement in component $j^{\text {th }}$ of color space.

$$
q^{j}=\operatorname{median}\left\{d^{j}\right\}
$$

iv-For the whole image, three quality numbers are available in each color space component, which is suggested to obtain a single number in the following equation.

$$
\mathbf{Q}=\sum_{j=1}^{3} w_{j} q^{j} ; \quad w_{1}+w_{2}+w_{3}=1
$$

Our suggestion for the weights of Eq. 7 is to consider the weight of the brightness component twice as much as the color components, ie: $w_{Y}=0.5, W_{C b}=0.25, w_{C r}=0.25$. However, in degradations which is focused on colors, $w_{Y}=$ $W_{C b}=w_{C r}=1 / 3$ also could be acceptable. Using this above procedure for comparison, measurement performance would be limited in range $[0,1]$ that smaller $Q$, better quality.

\footnotetext{
${ }^{2}$ let's consider $X$ and its estimated as $\hat{X}$ as two vector with $r$ element:if $e(m)=\left|\frac{\hat{x}(m)-x(m)}{x(m)}\right| \rightarrow \Sigma_{m=1}^{r} e(m) \leq \frac{\sum_{m=1}^{r}|x(m)-\hat{x}(m)|^{2}}{\sum_{m=1}^{r}|x(m)|^{2}}=$ $\frac{\|X-\hat{X}\|^{2}}{\|X\|^{2}}$. if $e(m)$ is greater than 1 in magnitude, then $\hat{x}$ can be considered erroneous, so we can represent all values larger than one that represent highly corrupted images with one.
} 
TABLE I: Two proposed methods abbreviations and required equations.

\begin{tabular}{|l|l|l|l|}
\hline symbol & definition & Equations & Number of required values \\
\hline$Q_{R}$ & $\begin{array}{l}\text { Median Quality of Reflected } \\
\text { coefficients in Rectangular path }\end{array}$ & Eqs.1,4,5,6,7 & $3 K r$ \\
\hline$Q_{D}$ & $\begin{array}{l}\text { Median Quality of Reflected } \\
\text { coefficients in Diagonal path }\end{array}$ & Eqs.3,2,4,5,6,7 & $3 K r$ \\
\hline
\end{tabular}

TABLE II: SROCC results of the proposed and two other methods on three databases.

\begin{tabular}{|l|l|l|l|l|l|}
\hline Database & Distortion & $Q_{R}$ & $Q_{D}$ & RRPSV & FMRP \\
\hline \multirow{4}{*}{ TID2013 } & AWGN & 0.8184 & 0.8369 & 0.8226 & 0.8214 \\
\cline { 2 - 6 } & BLUR & 0.9161 & 0.8902 & 0.8600 & 0.9296 \\
\cline { 2 - 6 } & JPEG & 0.7990 & 0.8064 & 0.8302 & 0.8934 \\
\cline { 2 - 6 } & JP2k & 0.8554 & 0.8292 & 0.8417 & 0.9254 \\
\hline \hline \multirow{4}{*}{ CSIQ } & AWGN & 0.7455 & 0.7900 & 0.8475 & 0.8865 \\
\cline { 2 - 6 } & BLUR & 0.9062 & 0.8684 & 0.8534 & 0.9090 \\
\cline { 2 - 6 } & JPEG & 0.9031 & 0.8908 & 0.8379 & 0.9185 \\
\cline { 2 - 6 } & JP2k & 0.9330 & 0.9178 & 0.8493 & 0.9330 \\
\hline \hline \multirow{3}{*}{ LIVE } & AWGN & 0.8226 & 0.8497 & 0.8519 & 0.9804 \\
\cline { 2 - 6 } & BLUR & 0.8195 & 0.8416 & 0.8346 & 0.9836 \\
\cline { 2 - 6 } & JPEG & 0.8384 & 0.8266 & 0.8201 & 0.9730 \\
\cline { 2 - 6 } & JP2K & 0.8458 & 0.8340 & 0.8359 & 0.9730 \\
\hline \hline \multicolumn{2}{|c|}{ required time of features(s) } & 0.065 & 0.065 & 0.19 & 0.99 \\
\hline
\end{tabular}

Eventually, two measurement methods were defined based on the extraction patterns. A summary of these methods and the required equations are listed in Table I, with the number of necessary values should be sent from a sender to the receiver. In following, the rank order correlation between subject qualification and objective measurement, Spearman Rank Order Correlation Coefficient (SROCC) as a suitable performance to compare experiment results of the proposed idea besides the other two methods, RRPSV [7] and FMRP [8] on three famous database LIVE [9], CSIQ [10] and TID2013 [11] would be presented in Table II. Article [7] compared the entropy of the reflective velues of the degraded and the corresponded reference image blocks in size $w \times w \times 3$ on the eigenvectors of covariance matrix of all the reference image blocks. Therefore, the time required in transmitter would be more than the receiver side. The eigenvectors matrix in size $w \times w \times 3$ should be transmitted besides one entropy value for each block. Article [8] is based on DT-CWT statistics and machine learning for regression. The required calculation time of feature vector for four methods are shown in the last row of Table II according to using a PC with $3.7 \mathrm{GHz}$ Intel Core i7 CPU and 16 GB RAM. Note that $Q_{R}$ and $Q_{D}$ methods applied only by calculating the difference without any processing, regression and learning. Here the purpose is just to ensure the efficiency of the vectors $R_{R}$ and $R_{D}$ empirically. Using entropy of each columns of feature matrix could identify the distribution of a frequency in the all image blocks and entropy of each row is the distribution frequency of each blocks.

2. Other application of these feature vectors could be evaluation of the complexity of patches as seen in Fig. 2 and 3 . If we can define a criterion for the pattern complexity of each image fragment, it will be possible to use different bit rates to compress the pieces to decreasing the amount of memory required to compress the whole image. Obtaining a numerical criterion to measure the complexity of images has always been considered [12] [13] [14] [15]. For this (a)

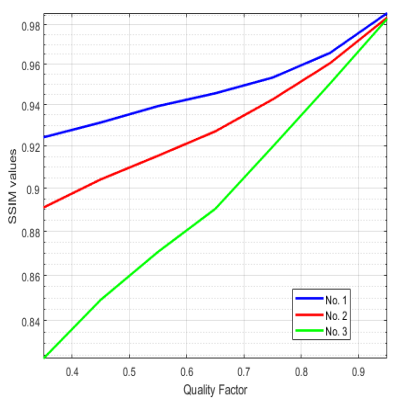

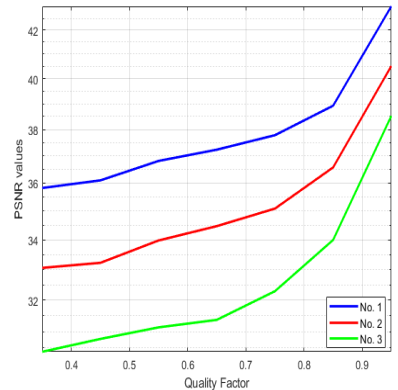

(b)
Fig. 5: (a) $S S I M$ and (b)PSNR of three blocks of Fig. 3 using different quality factor in jpeg compression.

purpose, it seems considering the brightness component $Y$ is sufficient to determining the complexity and the necessary quality parameter in jpeg compression. Although in this article, we do not seek to find the optimum of the threshold level or a criterion for clustering blocks, and just would like to show the potential ability of using the feature vector $R_{R}$. Let's consider three patches and coding them with quality factor of 0.35 and .45 and ... and .95 using 'imwrite' in Matlab in format jpeg. The values of PSNR and SSIM of the reconstruction patches, with respect to their main blocks are shown in Fig. 5. As shown, more crowded images require to use a higher quality parameter. The value of this complexity is simply calculated and related to Eq. 8 .

$$
s=1-\frac{\sum_{n=1}^{\frac{r}{2}} R_{R}^{Y}(n)}{\sum_{n=1}^{r} R_{R}^{Y}(n)}
$$

where $R_{R}^{Y}$ is the feature vector in component $Y$ in size $1 \times r$. Indeed, Eq. 8 shows what power of the image patch lies in low frequencies, the higher $s$, more complex areas. Complexity parameters of blocks are $s_{1}=0.2414, s_{2}=0.3414, s_{3}=$ 0.4338 and as seen in Figs. 5, larger complexity, larger quality factor required. Patch No.1 which is in uniform areas, could be compressed in smaller bit rate and simultaneously save quality image.

\section{CONCLUSION}

In the present article, a feature extraction layout of an image for further processing is introduced that integrates the properties' small size, with the necessary information besides simplicity and efficient computation. Since examining the indicators of a whole image at a glance, can not show its details well, so we used the common method of image blocking. DCT Transformation was the second stage of work, which is a good indicator for the image and doesnot required to transferring or storing transformation matrix. The size of the DCT block $r \times r$ in two ways from number $r^{2}$ to member $r$ values decreased. Experiments performed on two applications showed that without any additional processing, these feature vectors received confidence score to determine the quality of images in reduced reference mode and also identify the image complexity for using various quality factor to reduce bit rate after compression. 


\section{REFERENCES}

[1] A. Humeau-Heurtier, "Texture feature extraction methods: A survey," IEEE Access, vol. 7, pp. 8975-9000, 2019.

[2] Z. Liu, Z. Lai, W. Ou, K. Zhang, and R. Zheng, "Structured optimal graph based sparse feature extraction for semi-supervised learning," Signal Processing, vol. 170, pp. 107-456, 2020.

[3] W. Zhou, S. Gao, L. Zhang, and X. Lou, "Histogram of Oriented Gradients Feature Extraction from Raw Bayer Pattern Images," IEEE Transactions on Circuits and Systems II: Express Briefs, vol. 67, no. 5, pp. 946-950, 2020.

[4] A. O. Salau and S. Jain, "Feature Extraction: A Survey of the Types, Techniques, Applications," 2019 International Conference on Signal Processing and Communication, ICSC 2019, pp. 158-164, 2019.

[5] J. Wang, L. Wang, F. Nie, and S. Member, "Joint Feature Selection and Extraction With Sparse Unsupervised Projection," IEEE TRANSACTIONS ON NEURAL NETWORKS AND LEARNING SYSTEMS, pp. $1-11,2021$.

[6] A. Zarić, N. Tatalović, N. Brajković, H. Hlevnjak, M. Loncrić, E. Dumić, and S. Grgić, "VCL@FER-baza slika za procjenu kvalitete slike," Automatika, vol. 53, no. 4, pp. 344-354, 2012.

[7] F. Torkamani-Azar and J. Parkkinen, "Image quality assessment using block-based weighted SVD," Signal, Image and Video Processing, vol. 12 , no. 7, 2018.

[8] X. Xie, P. Carré, C. Perrine, Y. Pousset, N. Zhou, and J. Wu, "Reduced-reference image quality metric based on statistic model in complex wavelet transform domain," Signal Processing: Image Communication, vol. 74, no. January 2018, pp. 218-230, 2019. [Online]. Available: https://doi.org/10.1016/j.image.2019.02.006

[9] H. R. Sheikh, M. F. Sabir, and A. C. Bovik, "Image Quality Assessment Algorithms," Image Processing, IEEE Transactions on, vol. 15, no. 11, pp. 3441-3452, 2006.

[10] D. M. Chandler, "Most apparent distortion: full-reference image quality assessment and the role of strategy," Journal of Electronic Imaging, vol. 19, no. 1, 2010.

[11] N. Ponomarenko, L. Jin, O. Ieremeiev, V. Lukin, K. Egiazarian, J. Astola, B. Vozel, K. Chehdi, M. Carli, F. Battisti, and C.-C. Jay Kuo, "Image database tid2013: Peculiarities, results and perspectives," Signal Processing: Image Communication, vol. 30, pp. 57-77, 2015.

[12] I. Mario, M. Chacón, D. Alma, and S. Corral, "Image complexity measure: A human criterion free approach," Annual Conference of the North American Fuzzy Information Processing Society - NAFIPS, vol. 2005, pp. 241-246, 2005.

[13] E. Guldogan and M. Gabbouj, "Feature selection for content-based image retrieval," Signal, Image and Video Processing, vol. 2, no. 3, pp. 241-250, 2008.

[14] S. Amirshahi and F. T. Azar, "Human optic sensitivity computation based on singular value decomposition," Optica Applicata, vol. XLII, no. 1, 2012.

[15] S. E. Corchs, G. Ciocca, E. Bricolo, and F. Gasparini, "Predicting complexity perception of real world images," PLOS ONE, vol. 11, no. 6, pp. 1-22, 2016. 